\title{
Active Growth and Pattern Formation in Membrane-Protein Systems
}

\author{
F. Cagnetta, M. R. Evans, and D. Marenduzzo \\ SUPA, School of Physics and Astronomy, University of Edinburgh, \\ Peter Guthrie Tait Road, Edinburgh EH9 3FD, United Kingdom
}

(Received 23 October 2017; revised manuscript received 14 February 2018; published 18 June 2018)

\begin{abstract}
Inspired by recent experimental observations of patterning at the membrane of a living cell, we propose a generic model for the dynamics of a fluctuating interface driven by particlelike inclusions which stimulate its growth. We find that the coupling between interfacial and inclusions dynamics yields microphase separation and the self-organization of traveling waves. These patterns are strikingly similar to those detected in experiments on biological membranes. Our results further show that the active growth kinetics do not fall into the Kardar-Parisi-Zhang universality class for growing interfaces, displaying instead a novel superposition of scaling and sustained oscillations.
\end{abstract}

DOI: 10.1103/PhysRevLett.120.258001

Active membranes and interfaces have revealed fascinating complex pattern formation and nontrivial dynamical features [1-3]. An interface is termed active when its dynamics violate detailed balance due to the presence of local nonthermal forces. A paradigmatic example is that of the plasma membrane of an eukaryotic cell, which is driven far from equilibrium by its constant interaction with ion channels, membrane proteins, and the actin cytoskeletal network [4], all of which are intimately coupled to the membrane fluctuations $[5,6]$.

Recent experiments have unveiled a wide variety of organized dynamical structures formed within the plasma membrane of crawling cells. Membrane-binding proteins such as GTPases of the Rho and Ras families, for instance, form dynamic nanoclusters [7-9], while ripples develop on the membrane itself and surf as a traveling wave [10-12]. A generic picture accounting for the emergence of all these structures is still lacking. Could the fact that the proteins activate growth be the underlying cause of such a complex scenario?

In this work, we explore this possibility by introducing a minimal nonequilibrium model for pattern formation in a system of active inclusions embedded in an active interface. The feedback between such particlelike inclusions and the interfacial dynamics, rooted in experimental observations, assumes the membrane motion to be regulated by transmembrane proteins [15], which, in turn, are coupled to the membrane local shape [16]. The mechanism we identify relies on activity alone and dispenses with the need for nonlinear biochemistry as invoked previously in models assuming an underlying activator-inhibitor dynamics $[8,17]$. We also stress that the mechanism requires no assumption on the polar patterns which may be formed by the underlying actin cortex [10]: all that is required is polymerization normal to the surface. Besides being relevant to pattern formation on eukaryotic membranes, our model extends the problem of semiautonomous systems, such as randomly advected passive scalar fields [18] or passive sliders on fluctuating interfaces $[19,20]$, into the active matter realm.

First, we show that the inclusion-interface coupling provides a generic route to patterning along with microphase separation and waves. This is a general result, which does not depend on fine-tuning of model parameters. We further provide a simple theory based on the analysis of shock and rarefaction waves, which, on the one hand, correctly predicts the numerically observed scaling of cluster size and wave velocity with the model parameters, and, on the other hand, reveals the intimate connection between clustering, waves, and the underlying motion of the interface. Importantly, the feedback requires noise to be effective, as only damped waves survive in a mean-field deterministic framework (see, e.g., Ref. [21]). Second, our work suggests that an actively growing interface cannot be described by the Kardar-ParisiZhang (KPZ) equation [22], which successfully represents the universal features of the passive case. Instead, we find nontrivial sustained oscillations in the roughening dynamics, which could be the key signature to look for in future experiments with active membranes.

As the leading edge of a crawling cell is an essentially 1D object, we model the fluctuating interface as a directed random walk in $(1+1) \mathrm{D}$ (Fig. 1). The dynamics, as in standard models of stochastic growth, entail only local single-step moves [23]. Pictorially, the interface comprises positive and negative slopes / and $\backslash$ joining $L$ sites. Each downward kink $\vee$ transforms into an upward one $\wedge$ (and vice versa), at rate $p_{+}\left(p_{-}\right)$(see Fig. 1). The inclusions in the interface break detailed balance by stimulating interface growth (i.e., biasing its motion toward the top in Fig. 1). This is inspired by the upregulation of actin polymerization due to Rho GTPases such as Rac1 and Cdc42 [15]. Note, however, that we are not considering any specific function 


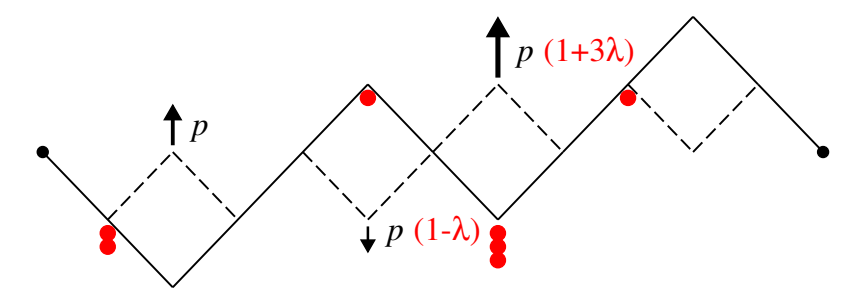

FIG. 1. Schematics of our active interface- (black solid line) inclusions (red circles) model. Dashed lines denote the moves defining our interface updating rule. The detailed-balance breaking action of the inclusions enhances the growth rate (thicker upward arrow) and hampers the reverse move (thinner downward arrow) proportionally to the local number of inclusions.

(except growth stimulation) or shape for the inclusions, as done, for instance, in Ref. [6] with asymmetric pumps. We set

$$
p_{ \pm}=p\left(1 \pm \lambda n_{i}\right)
$$

where $n_{i}$ is the number of inclusions at the $i$ th site. With this choice, growth is favored on occupied sites, as $p_{+}-p_{-} \propto \lambda n_{i}$, and we can control its strength by varying $\lambda \geq 0$. Setting $\lambda \neq 0$ is the key ingredient that makes our interface active and our problem different from the semiautonomous systems cited above.

Additionally, $N$ inclusions diffuse and are advected by the interfacial slope, mimicking the coupling of protein transport to local surface curvature observed for several membrane-binding proteins $[16,21]$. We consider a "curvophobic" coupling, where proteins tend to drift toward regions of negative curvature. Each inclusion jumps independently left or right with rates $q_{+}$and $q_{-}$,

$$
q_{ \pm}=q\left(1 \pm \frac{\gamma}{2} \nabla h_{i}\right)
$$

where $\nabla h_{i}=\left(h_{i+1}-h_{i-1}\right) / a$, so that $\gamma$ measures the strength of the slope-mediated advection. We highlight here that the feedback between inclusions and interface dynamics is realized only when both $\lambda$ and $\gamma$ are greater than 0 , making $\gamma$ a key ingredient of our model. We set the unbiased rates $p$ and $q$ (obtained when $\gamma=\lambda=0$ ) to 1 , implying comparable time scales of inclusion and interface dynamics, and the global particle concentration to 1 (i.e., $N=L$ ). As explained in the Supplemental Material [24], the specific values of such parameters do not alter the physics of the system unless pushed to extreme values. This set of update rules augmented with periodic boundary conditions leads to stochastic dynamics for the active interface-inclusions system.

Figure 2 shows typical snapshots of the interface profile and inclusion distribution as a function of time, when $\gamma$ and $\lambda$ are both strictly positive (for more details of the simulation methods, see the Supplemental Material [24]).

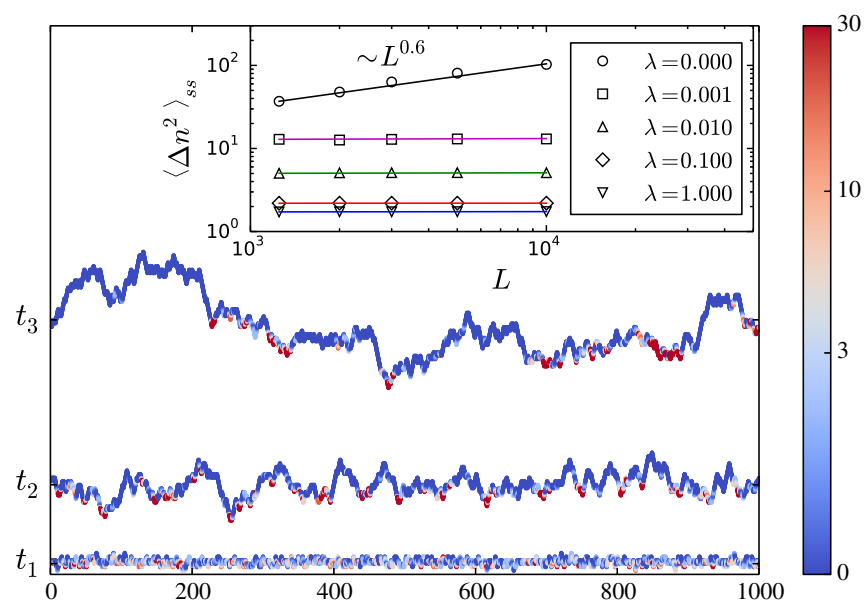

FIG. 2. Snapshots of a fluctuating interface of size $L=1000$ with $N=L$ inclusions, at three different times $t_{1}<t_{2}<t_{3}$. As time passes, the membrane roughens and particles form clusters marked by the red spots on the interface profile. Here, $\gamma=1$ while $\lambda=0.01$ so as to enhance visibility of clusters, but the same scenario is observed for each value of $\gamma, \lambda>0$. The inset shows the inclusions number variance [average of $\left(n_{i}-L^{-1} \sum_{j=1}^{L} n_{j}\right)^{2}$ over several realizations of the noise] in steady state, together with the $\lambda=0$ limit. The absence of scaling with system size $L$ for $\lambda>0$ signals arrested coarsening and microphase separation.

Initially, the surface is flat and inclusions are uniformly distributed (bottom snapshot). Later on, the interface roughens and inclusions accumulate in valleys (center and top snapshots). They do so since $\gamma>0$ favors advection toward regions with negative curvature (valleys). Interestingly, clusters are also strongly affected by $\lambda$. In the $\lambda \rightarrow 0$ limit, our model reduces to the passive problem considered in Ref. [26], where particles slide on an equilibrium fluctuating interface. In this limit, the density fluctuations grow in time so as to reach a steady state, scaling with the system size $L$ as $L^{0.6}$ (Fig. 2, inset), consistent with numerical predictions [26]. Notably, as soon as active growth is turned on $(\lambda>0)$, we find a completely different scenario in which the steady-state density fluctuations no longer scale with $L$ (Fig. 2, inset).

It is also useful to compare our results to those obtained in Refs. [19,20,27], where particles slide either on a KPZ or equilibrium interface. The former case corresponds to the limit $\lambda n_{i} \rightarrow \lambda$ of Eq. (1), which removes the local concentration dependence in the interface dynamics. In both these passive cases, inclusions aggregate in interface valleys in a fluctuating fashion due to the noise-induced flipping of valleys. As a result of this phase separation, the steady-state density fluctuations scale as a power of the system size $L$. Conversely, the absence of scaling we observe means that the clusters reach a self-limiting size. In other words, the active growth term $\lambda n_{i}$ leads to noisy microphase (rather than macrophase) separation. The mechanism underlying cluster formation is that advection promotes particle congregation in valleys. The clustering cannot proceed 
indefinitely, however, as inclusions stimulate the growth of a local "bump" in the interface, which eventually drives them away, arresting coarsening. The higher $\lambda$ is, the sooner we expect the cluster to disperse, and the smaller its size: this is what we find numerically. Intriguingly, clustering requires thermal fluctuations; a mean-field deterministic description of our model leads to an advancing flat interface with no patterning at late times.

We now turn to the dynamics in the microphase separated state. The argument above suggests that clusters tend to move away from the bumps they generate. Remarkably, these small aggregates self-organize into traveling waves accompanied by membrane ripples. The resulting membrane waves are readily visible in the kymograph in Fig. 3. The traveling waves in the inclusion density profiles are shown in Fig. 3, top panel, and a sketching of the mechanism for wave generation is shown in the bottom panel. When $\lambda>0$, a cluster of inclusions creates a bulge in the interface (left side of panel). Since $\gamma>0$, inclusions will be pushed to the two regions of negative curvature on the sides of the bump (center of panel) and generate new bumps resulting in the lateral spreading of membrane protrusion (right side of the panel). To understand quantitatively how wave speed and cluster size scale with $\gamma$ and $\lambda$,
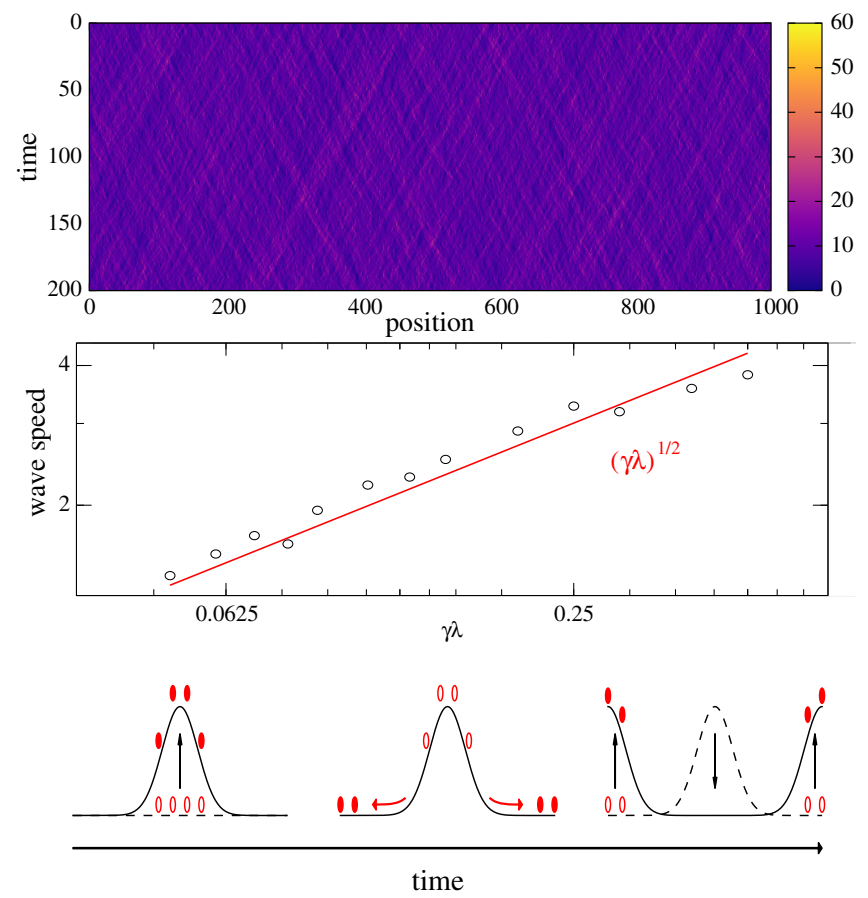

FIG. 3. Top: Kymograph of the inclusion density (represented by color) in a portion of an $L=N=10000$ system, $\lambda=\gamma=0.3$ in steady state. The light colored (red) lines indicate the lateral traveling density waves described in the text. Middle: The waves' speed is measured by the slope of these lines and plotted against $\lambda \gamma$ to match the prediction of our large-scale theory (see text). Bottom: Pictorial representation of wave generation in our model. Each sketch depicts the current (solid line, filled circles) and previous (dashed line, empty circles) configuration. we consider a large-scale description of the system obtained by a suitable coarse graining of the interface profile and inclusion density. This analysis is inspired by the theory of shallow water waves [28]. Using a standard procedure (see the Supplemental Material [24]), one may derive the following stochastic partial differential equations for the coarse-grained inclusion density field $n(x, t)$ and interface height $h(x, t)$,

$$
\begin{aligned}
& \partial_{t} n=\gamma \partial_{x}\left(n \partial_{x} h\right)+a \partial_{x}^{2} n+\xi_{c}, \\
& \partial_{t} h=\lambda n\left[1-\left(\partial_{x} h\right)^{2}\right]+a \partial_{x}^{2} h+\eta,
\end{aligned}
$$

where $a$ is the lattice spacing of the microscopic model. Both $\xi_{c}$ and $\eta$ in Eq. (3) are Gaussian, whereas $\xi_{c}$ is the divergence of a random current, so as to ensure conservation of the number of inclusions. Note that these equations can be deduced on general symmetry grounds at the price of losing the relation between the coefficients and the microscopic model parameters [29]. The active terms in the height equation are those controlled by the inclusion density $n(x, t)$ at variance with early models such as Ref. [1] where activity enters as colored noise.

Clusters and traveling waves emerge as shock solutions in the inviscid limit $(a \rightarrow 0)$ of the deterministic version of Eq. (3). By introducing the slope variable $u \equiv \partial_{x} h$, Eq. (3) acquires the structure of a hyperbolic set of conservation laws [30],

$$
\partial_{t}\left(\begin{array}{l}
n \\
u
\end{array}\right)+\partial_{x}\left(\begin{array}{c}
-\gamma n u \\
-\lambda n
\end{array}\right) \equiv \partial_{t} \mathbf{v}+\partial_{x} \mathbf{f}(\mathbf{v})=0
$$

where we introduced a vectorial notation and further neglected the KPZ nonlinearity, so as to highlight that our patterns are generated by activity alone. We will show that neglecting the KPZ term gives reasonable results, although its relevance for other aspects of the model is an open question. We call $\mathbf{F}$ the matrix with elements $F_{\mu \nu}=\partial f_{\mu} / \partial v_{\nu}$, and $\zeta_{\mu}, \mathbf{r}_{\mu}$ its $\mathbf{v}$-dependent eigenvalues, and corresponding right eigenvectors $(\mu, \nu=1,2)$. For each positive value of $\gamma$ and $\lambda, \mathbf{F}$ obeys the genuine nonlinearity condition $\left(\partial \zeta_{\mu} / \partial \mathbf{v}\right) \cdot \mathbf{r}_{\mu}>0$ [30]. As a consequence, Eq. (4) admits rarefaction fan and shock wave solutions in the whole $\lambda, \gamma>0$ range of parameters. Such solutions can be explicitly obtained by studying the corresponding Riemann problem, i.e., Eq. (4) on an infinite domain with a Heaviside-function initial condition $\mathbf{v}=\mathbf{v}_{l}$ for $x<0, \mathbf{v}_{r}$ for $x>0$, then using the outcomes as building blocks for the full problem. In a shock wave, for instance, the initial discontinuity travels ballistically with a fixed speed $\sigma$ depending on initial state, as well as $\gamma$ and $\lambda$.

Two conditions are required for a shock wave to develop. One is the Rankine-Hugoniot condition relating the wave speed to the currents across the shock front, 


$$
\sigma[[\mathbf{v}]]=[[\mathbf{f}(\mathbf{v})]]
$$

where [[.]] denotes the size of the discontinuity across the shock. The other is the requirement that the interfacial slope on the right of the shock wave is higher than that on the left, as shock waves arise within valleys. A representative case is the evolution of a valley uniformly filled with particles, $u_{l}=-1, u_{r}=1, n_{l}=n_{r}=1$. The slope and density fields at later times are given by [31]

$$
(u(x, t), n(x, t))= \begin{cases}(-1,1), & x<-\sqrt{\gamma \lambda} t, \\ (0,1+\sqrt{\gamma / \lambda}), & |x|<\sqrt{\gamma \lambda} t \\ (1,1), & x>\sqrt{\gamma \lambda} t\end{cases}
$$

so that a typical wave speed $\sigma$ is readily identified as the shock speed, and we can interpret a typical cluster size $s$ as the excess density of the cluster

$$
\sigma \sim \sqrt{\gamma \lambda} ; \quad s \sim \sqrt{\gamma / \lambda} .
$$

Remarkably, the predicted scaling (6) captures that seen numerically (Fig. 3, top panel, and Fig. 4). The scaling of the cluster size $s(6)$ explains why the case $\lambda \neq 0$ leading to microphase separation and clustering is fundamentally different from the singular passive limit [10], where cluster size diverges. Additionally, our theory suggests that all systems with nonzero $\lambda$ and $\gamma$ display equivalent features. Notably, these deterministic shock waves decay diffusively as soon as $a \neq 0$ [30], so that noise is required to sustain them in steady state by continuously generating kinks which create further shocks.

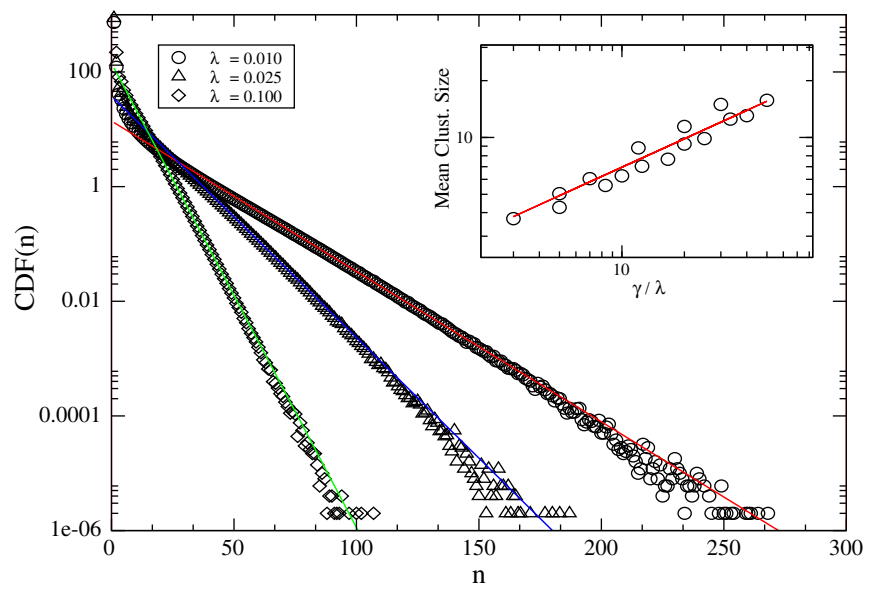

FIG. 4. Semilog plot of the cluster distribution function (CDF) of protein clusters for an $L=N=5000$ system, $\gamma=1.0$ and $\lambda$ as in the key. The exponential tail implies a typical cluster size, which does not vary with $L$ : this is the hallmark of microphase separation. In the inset, this typical size is plotted against $\gamma / \lambda$ together with the prediction of our theory (red line), showing again remarkable agreement.
Finally, we measured the interface width

$$
w^{2}(L, t)=\frac{1}{L}\left\langle\int_{0}^{L} d x\left(h(x, t)-\frac{1}{L} \int_{0}^{L} d x h(x, t)\right)^{2}\right\rangle .
$$

The initial growth of $w^{2}(L, t)$ defines the exponent $\beta$ via $w(L, t) \sim t^{\beta}$, whereas the steady-state value $w_{\mathrm{SS}}(L)$ defines the exponent $\alpha$ through $w_{\mathrm{SS}}(L) \sim L^{\alpha}$, with $L$ the system size. If $\lambda=0$, the interface dynamics decouples from the inclusions, and its width grows as in the EdwardsWilkinson (EW) model, with $\beta=1 / 4$ and $\alpha=1 / 2$. If $\lambda \neq 0$ and the protein density is uniform, the dynamics is described by the KPZ scaling, $\beta=1 / 3$ and $\alpha=1 / 2$. Intriguingly, the growth of our active interface reverts to an EW growth law for the width but with sustained oscillations superposed (Fig. 5).

The width oscillations we observe are a novel phenomenon, intimately coupled to the underlying wavelike dynamics of the inclusions. When clusters form, the interface growth is dominated by the inhomogeneous active contribution; hence, it is faster than in the noise-driven passive (EW) case [1]. This corresponds to the rising curve of the oscillation. Once clusters start to move, their associated ripples surf the interface and progressively smoothen it. This results in a significant decrease in the width. Dimensional analysis suggests that the oscillation period should scale as the ratio between a length scale and the shock wave speed. Our numerics confirm this and show the length scale to be the interface length $L$ (Fig. 5, inset). This implies that clusters move a finite fraction of the whole system independently of their size. Strikingly, simulations also suggest that the oscillation period is of the same order of the time for microphase separation to occur (measured through the saturation of density fluctuations), supporting the idea that the latter takes place through wave collisions.

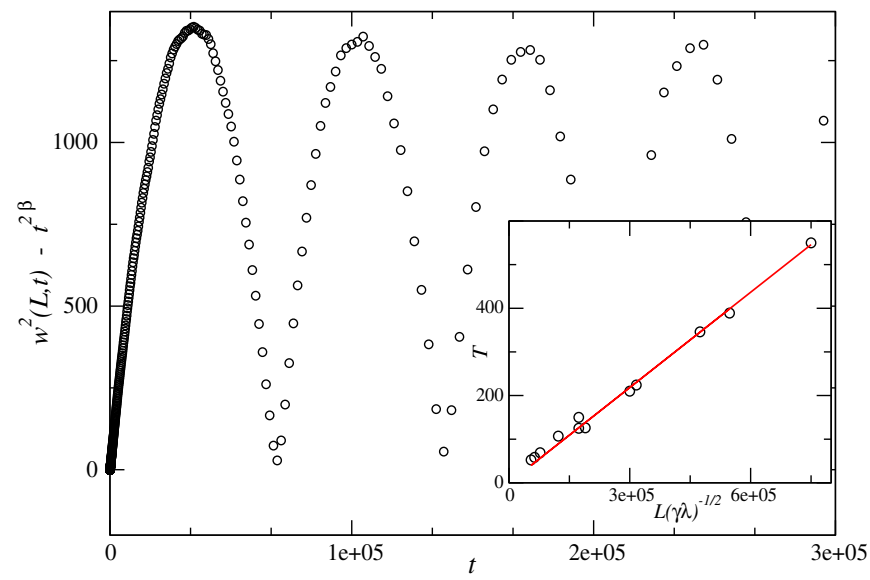

FIG. 5. Oscillating component of the squared interface width for an $L=N=30000$ system, $\lambda=0.6$ and $\gamma=0.25$. Nonlinear oscillations are manifest once the Edwards-Wilkinson term $t^{2 \beta}$, $\beta \simeq 1 / 4$ is subtracted. The inset shows the dependence of the period on model parameters and system size. 
To conclude, we have shown how minimal feedback between a system of particles and a growing interface can lead to spatiotemporal patterns reminiscent of membrane waves [8,9] and protein nanoclusters [10,32]. The mechanism relies on interfacial noise, which, by generating kinks in the interface profile, seeds an inclusion cluster, which in turn, produces a kinematic wave due to feedback between inclusion and interface dynamics. Furthermore, we have found the active interface roughening to consist of a scaleinvariant component well described in 1D by EdwardWilkinson exponents but with superposed oscillations whose features are determined by the collective behavior of the system components. Our theory predicts scaling relations (6) for the features of the kinematic waves and microphase separation which can be experimentally checked, provided an estimate of $\lambda$ and $\gamma$ can be made [33]. To what extent such features are retained in $2 \mathrm{D}$ is a question to be addressed in future work. Preliminary results of an extension of our stochastic dynamics to 2D (see the Supplemental Material [24] for details) do suggest the occurrence of microphase separation. However, 2D affords a variety of extensions of the model due to the tensorial nature of curvature, and it would be of interest to explore further the different possibilities.

F. C. acknowledges support from Scottish Funding Council under a studentship. D. M. and M. R. E. acknowledge funding under Engineering and Physical Sciences Research Council Grant No. EP/J007404/1.

[1] J. Prost and R. Bruinsma, Europhys. Lett. 33, 321 (1996).

[2] S. Ramaswamy and M. Rao, C. R. Acad. Sci. Ser. Gen. Ser., 2, 817 (2001).

[3] A. Maitra, P. Srivastava, M. Rao, and S. Ramaswamy, Phys. Rev. Lett. 112, 258101 (2014).

[4] D. Bray, Cell Movements: From Molecules to Motility (Garland Science, New York, 2001).

[5] J.-B. Manneville, P. Bassereau, D. Levy, and J. Prost, Phys. Rev. Lett. 82, 4356 (1999).

[6] S. Ramaswamy, J. Toner, and J. Prost, Phys. Rev. Lett. 84, 3494 (2000).

[7] A. B. Goryachev and A. V. Pokhilko, FEBS Lett. 582, 1437 (2008).

[8] W. M. Bement, M. Leda, A. M. Moe, M. A. Kita, M. E. Larson, A. E. Golding, C. Pfeuti, K.-C. Su, A. L. Miller, A. B. Goryachev et al., Nat. Cell Biol. 17, 1471 (2015).

[9] A. B. Goryachev, M. Leda, A. L. Miller, G. von Dassow, and W. M. Bement, Small GTPases 7, 65 (2016).

[10] K. Gowrishankar, S. Ghosh, S. Saha, C. Rumamol, S. Mayor, and M. Rao, Cell 149, 1353 (2012).

[11] J. Allard and A. Mogilner, Curr. Opin. Cell Biol. 25, 107 (2013).
[12] Such waves are confined to the membrane leading edge; hence, they are of a fundamentally different nature than the polarized subcellular actin waves observed in cells recovering from massive depolymerization of their actin networks $[13,14]$.

[13] G. Gerisch, T. Bretschneider, A. Müller-Taubenberger, E. Simmeth, M. Ecke, S. Diez, and K. Anderson, Biophys. J. 87, 3493 (2004).

[14] T. Le Goff, B. Liebchen, and D. Marenduzzo, Phys. Rev. Lett. 117, 238002 (2016).

[15] A. Hall, Science 279, 509 (1998).

[16] R. Lenarcic, S. Halbedel, L. Visser, M. Shaw, L. J. Wu, J. Errington, D. Marenduzzo, and L. W. Hamoen, EMBO J. 28, 2272 (2009).

[17] G. L. Ryan, H. M. Petroccia, N. Watanabe, and D. Vavylonis, Biophys. J. 102, 1493 (2012).

[18] R. H. Kraichnan, Phys. Rev. Lett. 72, 1016 (1994).

[19] D. Das and M. Barma, Phys. Rev. Lett. 85, 1602 (2000).

[20] D. Das, M. Barma, and S. N. Majumdar, Phys. Rev. E 64, 046126 (2001).

[21] N. S. Gov and A. Gopinathan, Biophys. J. 90, 454 (2006).

[22] M. Kardar, G. Parisi, and Y.-C. Zhang, Phys. Rev. Lett. 56, 889 (1986).

[23] M. Plischke, Z. Rácz, and D. Liu, Phys. Rev. B 35, 3485 (1987).

[24] See Supplemental Material at http://link.aps.org/ supplemental/10.1103/PhysRevLett.120.258001 for details of the simulations, a derivation of the coarse-grained equation and numerical results on a $(2+1) \mathrm{D}$ version of the model, which includes Ref. [25].

[25] B. M. Forrest and L.-H. Tang, Phys. Rev. Lett. 64, 1405 (1990).

[26] M. Gopalakrishnan, Phys. Rev. E 69, 011105 (2004).

[27] A. Nagar, M. Barma, and S. N. Majumdar, Phys. Rev. Lett. 94, 240601 (2005).

[28] H. Holden and N. H. Risebro, Front Tracking for Hyperbolic Conservation Laws (Springer, New York, 2015), Vol. 152.

[29] Specifically, one should ask for space-time translational invariance, invariance with respect to $x \leftrightarrow-x, n$ to be conserved, and the interface up-down symmetry to be broken only where $n \neq 0$.

[30] P. D. Lax, Hyperbolic Systems of Conservation Laws (SIAM, Philadelphia, 1973).

[31] The global conservation of inclusion number is seemingly lost here, as Eq. (5) solves Eq. (4) only on an infinite domain. However, conservation is readily restored, e.g., by addition of PBC.

[32] A. Chaudhuri, B. Bhattacharya, K. Gowrishankar, S. Mayor, and M. Rao, Proc. Natl. Acad. Sci. U.S.A. 108, 14825 (2011).

[33] While $\lambda$ is linked to the growth rate and should be measurable from the average lamellipodium speed, an estimate for $\gamma$ could be obtained from comparing the observed protein diffusivity to a bare one corresponding to purely thermal motion. 